\title{
Cavernoma-related epilepsy in cavernous malformations located within the temporal lobe: surgical management and seizure outcome
}

\author{
Patrick Schuss, MD, ${ }^{1}$ Julia Marx, ${ }^{1}$ Valeri Borger, MD, ${ }^{1}$ Simon Brandecker, MD, Ági Güresir, MD, ${ }^{1}$ \\ Alexis Hadjiathanasiou, MD, ${ }^{1}$ Motaz Hamed, MD, ${ }^{1}$ Matthias Schneider, MD, ${ }^{1}$ Rainer Surges, MD, ${ }^{2}$ \\ Hartmut Vatter, MD, ${ }^{1}$ and Erdem Güresir, MD' ${ }^{1}$
}

Departments of ${ }^{1}$ Neurosurgery and ${ }^{2}$ Epileptology, Rheinische Friedrich-Wilhelms-University, Bonn, Germany

\begin{abstract}
OBJECTIVE Cavernoma-related epilepsy (CRE) is a frequent symptom in patients with cerebral cavernous malformations (CCMs). Reports on surgical management and seizure outcome of epileptogenic CCM often focus on intracranial cavernoma in general. Therefore, data on CCMs within the temporal lobe are scarce. The authors therefore analyzed their institutional data.
\end{abstract}

METHODS From 2003 to 2018, 52 patients suffering from CCMs located within the temporal lobe underwent surgery for CRE at University Hospital Bonn. Information on patient characteristics, preoperative seizure history, preoperative evaluation, surgical strategies, postoperative complications, and seizure outcome was assessed and further analyzed. Seizure outcome was assessed 12 months after surgery according to the International League Against Epilepsy (ILAE) classification and stratified into favorable (ILAE class I) versus unfavorable (ILAE classes II-VI).

RESULTS Overall, 47 (90\%) of 52 patients with CCMs located in the temporal lobe and CRE achieved favorable seizure outcome. Pure lesionectomy was performed in 5 patients, extended lesionectomy with resection of the hemosiderin rim in 38 patients, and anterior temporal lobectomy in 9 patients with temporal lobe CCM. Specifically, 36 patients (69\%) suffered from drug-resistant epilepsy (DRE), 3 patients (6\%) from chronic CRE, and 13 patients (25\%) sustained sporadic CRE. In patients with DRE, favorable seizure outcome was achieved in $32(89 \%)$ of 36 patients. Patients with DRE were significantly older than patients with CCM-associated chronic or sporadic seizures $(p=0.02)$. Furthermore, patients with DRE more often underwent additional amygdalohippocampectomy following the recommendation of presurgical epileptological evaluation.

CONCLUSIONS Favorable seizure outcome is achievable in a substantial number of patients with epileptogenic CCM located in the temporal lobe, even if patients suffered from drug-resistant CRE. For adequate counseling and monitoring, patients with CRE should undergo a thorough pre- and postsurgical evaluation in dedicated epilepsy surgery programs.

https://thejns.org/doi/abs/10.3171/2020.1.FOCUS19920

KEYWORDS epilepsy surgery; seizure outcome; temporal cavernous malformation

$\mathrm{C}$ EREBRAL cavernous malformations (CCMs) are angiographically occult vascular lesions found in approximately $0.5 \%$ of the population. ${ }^{4} \mathrm{CCMs}$ nowadays are predominantly diagnosed in patients undergoing cerebral workup for multiple reasons such as trauma, stroke, or other diseases. ${ }^{10,18}$ In addition to the possibility of causing intracranial hemorrhage, cavernoma-related epilepsy (CRE) is a common initial symptom of supratentorial CCM. ${ }^{11}$ Therefore, resective epilepsy surgery is an established treatment option. ${ }^{4,13}$ Several studies have investigated the influence of the surgical strategies (such as resection of the hemosiderin rim) on postoperative seizure outcome in patients with CCM-related epilepsy. ${ }^{5,11,12,19} \mathrm{~A}$ large number of patients with CCM-related epilepsy present with the triggering cavernous lesion located within the temporal lobe, which is considered to be highly epileptogenic. ${ }^{12}$ Data on the surgical management and seizure outcome in patients with CCMs located within the temporal

ABBREVIATIONS CCM = cerebral cavernous malformation; CRE = cavernoma-related epilepsy; DRE = drug-resistant epilepsy; ILAE = International League Against Epilepsy.

SUBMITTED November 30, 2019. ACCEPTED January 23, 2020.

INCLUDE WHEN CITING DOI: 10.3171/2020.1.FOCUS19920. 
lobe only, and CRE, are scarce. Therefore, the purpose of the present study was to assess the institutional data from the Bonn epilepsy and neurovascular surgery program in order to analyze surgical management of patients with $\mathrm{CRE}$ and CCMs located solely in the temporal lobe.

\section{Methods \\ Patients}

Between 2003 and 2018, 52 patients suffering from CCM-related epilepsy with CCMs located precisely in the temporal lobe were treated in our neurosurgical department. Information on patient characteristics, preoperative seizure history, preoperative evaluation, surgical strategies, postoperative complications, and seizure outcome was obtained from our prospectively conducted epilepsy and neurovascular surgery database and further analyzed retrospectively. Establishment of these databases, as well as the present study, was approved by the local ethics committee.

\section{Type of Epilepsy}

Patients with temporal lobe CCMs and CCM-related single or multiple seizures during 1 year were categorized as having sporadic seizures. Drug-resistant epilepsy (DRE) was defined as having seizures for a minimum duration of 2 years despite adequate treatment with at least 2 different, appropriate antiepileptic drugs. Patients with persistent seizures without meeting the criteria for DRE were categorized as having chronic epilepsy.

\section{Presurgical Workup}

Patients with CCMs and DRE were assessed before surgery in the department of epileptology and were considered to be suitable for surgery. The standardized evaluation included detailed history of seizures, medical history, neuropsychological examination, high-resolution structural 3.0-T MRI, and video EEG monitoring using continuous recordings. Following a completed evaluation, an epileptological-based decision on the extent of resection was determined in every individual candidate by the interdisciplinary epilepsy conference.

Patients with CCMs and sporadic seizures also underwent standardized preoperative evaluation including detailed history of seizures, medical history, and highresolution structural 3.0-T MRI. Following a completed evaluation, a neurovascular-based indication and surgical resection strategy was determined in every individual patient by the interdisciplinary neurovascular conference.

\section{Surgical Management}

For further analysis, resection strategies were divided into pure lesionectomy (hemosiderin rim was not, or not completely, removed; Fig. 1A and B), extended lesionectomy (with hemosiderin rim; Fig. 1C and D), and anterior temporal lobectomy (Fig. 1E and F). Furthermore, in selected cases CCM resection was performed with additional amygdalohippocampectomy due to location of the temporal lobe CCM in the mesial temporal lobe structures or due to additional suspected mesial temporal sclerosis
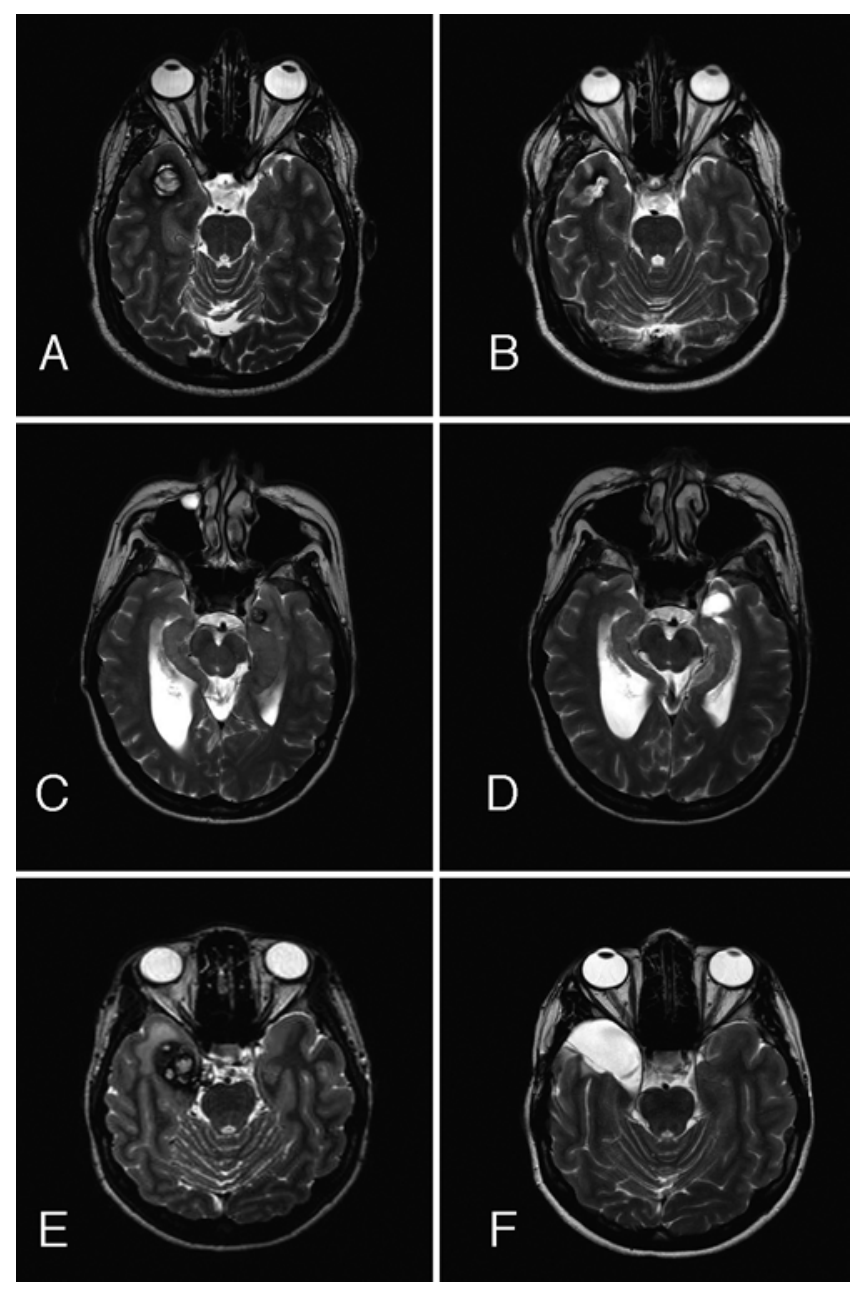

FIG. 1. Pre- and postsurgical axial T2-weighted MR images demonstrating the surgical strategies for epileptogenic CCM located within the temporal lobe. A and B: Pure lesionectomy. C and D: Extended lesionectomy (including the hemosiderin rim). $\mathrm{E}$ and F: Anterior temporal lobectomy.

as a potential secondary focus of epileptogenesis. Surgical management was conducted following the results and recommendations of the presurgical epileptological workup. The diagnosis of CCM was proven in all obtained surgical biopsy specimens by histopathological analysis.

\section{Seizure Outcome}

Seizure outcome was assessed 12 months after surgery according to the International League Against Epilepsy (ILAE) classification and stratified into favorable (ILAE class I) versus unfavorable (ILAE classes II-VI).

\section{Statistical Analysis}

Data analyses were performed using the computer software package SPSS (version 25, IBM Corp.). An unpaired t-test was used for parametric statistics. Categorical variables were analyzed in contingency tables using Fisher's exact test. Results with p values $<0.05$ were considered statistically significant. 


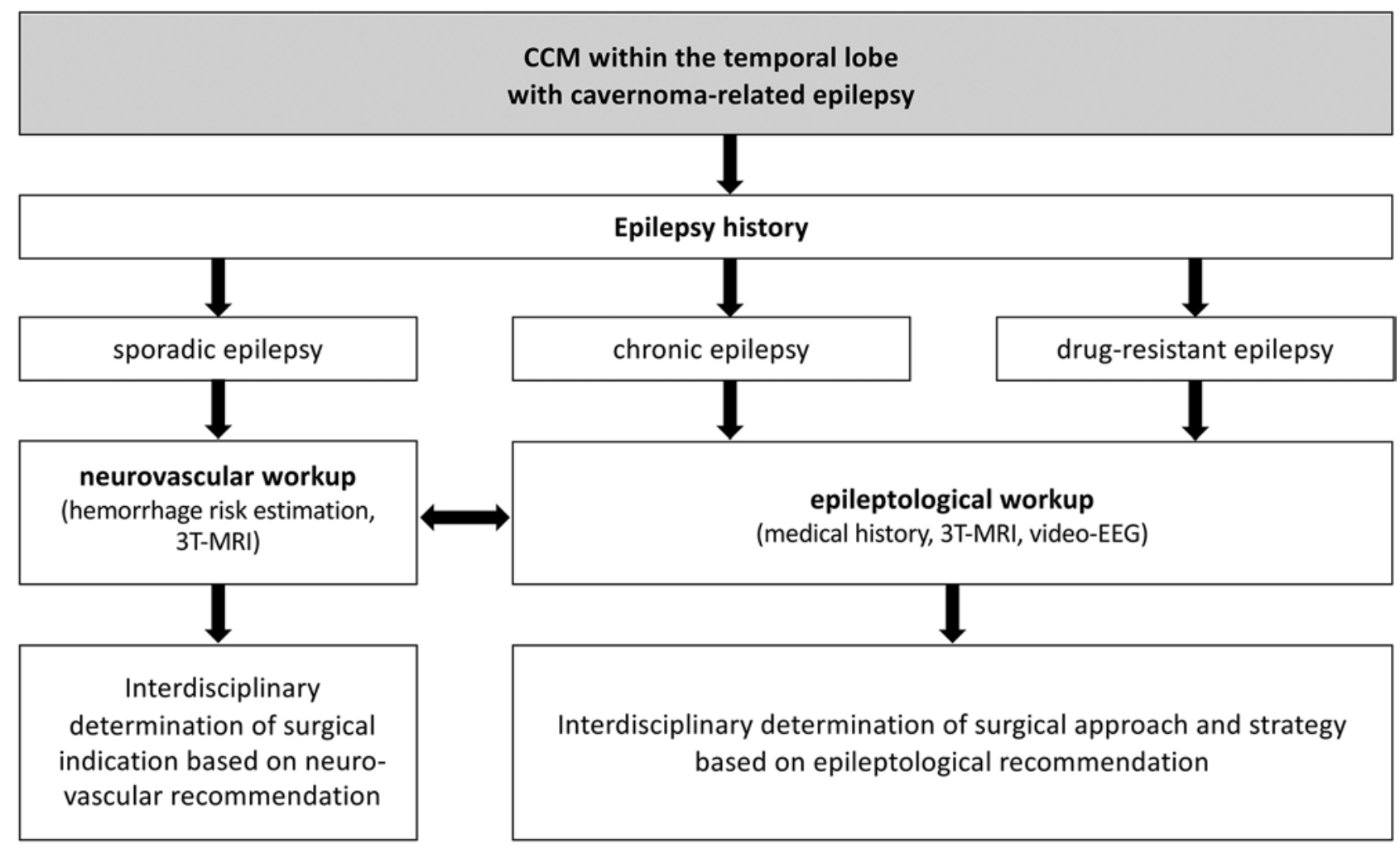

FIG. 2. Institutional workflow in patients with CCM within the temporal lobe, and CRE.

\section{Results}

\section{Patient Characteristics}

From 2003 to 2018, 52 patients with CCMs located within the temporal lobe were referred for surgical therapy to our tertiary epilepsy and neurovascular surgery center. All patients underwent epileptological and/or neurovascular presurgical workup depending on the purpose of their referral (Fig. 2). Details on patient characteristics are shown in Table 1.

\section{Surgical Management and Seizure Outcome of Temporal Lobe CCMs With CRE \\ Sporadic and Chronic Epilepsy}

Overall, 16 patients $(31 \%)$ suffered from sporadic or chronic epilepsy related to temporal lobe CCM at the time of their presentation. The mean age of patients with sporadic or chronic epilepsy was $38 \pm 13$ years. Patients with chronic epilepsy suffered for a mean of $29 \pm 15$ months before surgical treatment. Two patients with chronic epilepsy suffered from complex partial seizures, whereas 1 patient suffered from other focal seizures. None of the patients with chronic epilepsy and temporal lobe CCM suffered from secondarily generalized seizures. The mean size of the temporal lobe CCM was $15.7 \pm 8.2 \mathrm{~mm}$ in patients with sporadic or chronic epilepsy. Temporal lobe CCM was located medially in 7 patients suffering from CCM-related sporadic or chronic epilepsy and laterally in 9 patients. In this subgroup, 3 patients (19\%) underwent pure lesionectomy, 11 patients (69\%) underwent extended lesionectomy, and 2 patients (12\%) underwent anterior temporal lobectomy. Additional amygdalohippocampectomy was performed in 3 patients $(19 \%)$ suffering from sporadic or chronic epilepsy due to CCMs located within the temporal lobe. Specifically, additional amygdalohippocampectomy was performed in 2 patients with sporadic or chronic epilepsy due to location of the CCM in the mesial temporal lobe structures, and in 1 patient due to the diagnosis of additional mesial temporal sclerosis as a potential secondary focus of epilepsy. In all 3 patients with additional amygdalohippocampectomy, pre- and postoperative neuropsychological assessment was obtained. One of 3 patients had impaired baseline memory functions (verbal and nonverbal), which were stable postoperatively.

Overall, 15 (94\%) of 16 patients who suffered from sporadic or chronic epilepsy related to temporal lobe CCM achieved excellent seizure outcome (ILAE class I).

\section{Drug-Resistant Epilepsy}

Overall, 36 patients $(69 \%)$ suffered from DRE related to temporal lobe CCM. The mean age of patients with DRE was $49 \pm 17$ years. Patients with temporal lobe CCM and DRE were significantly older compared to patients with temporal lobe CCM and sporadic or chronic epilepsy $(\mathrm{p}=0.02,95 \%$ confidence interval 2.3-21.2). Three (8\%) of 36 patients who suffered from DRE related to temporal lobe CCM had secondarily generalized seizures as part of their DRE. The mean size of the temporal lobe CCM was $13.8 \pm 7.8 \mathrm{~mm}$ in patients with DRE. The temporal lobe CCM was located medially in 18 patients suffering 


\begin{tabular}{|c|c|}
\hline Variable & Value \\
\hline No. of patients & 52 \\
\hline Mean age $\pm S D$, yrs & $46 \pm 16$ \\
\hline Mean size of $\mathrm{CCM} \pm \mathrm{SD}, \mathrm{mm}$ & $14.3 \pm 7.9$ \\
\hline Female sex, $\mathrm{n}(\%)$ & $21(40)$ \\
\hline \multicolumn{2}{|l|}{ Type of epilepsy, n (\%) } \\
\hline Sporadic & $13(25)$ \\
\hline Chronic & $3(6)$ \\
\hline DRE & $36(69)$ \\
\hline \multicolumn{2}{|l|}{ Seizure semiology, $n(\%)$} \\
\hline Complex partial seizures & $27(52)$ \\
\hline Other focal seizures & $15(29)$ \\
\hline Generalized convulsions & $4(8)$ \\
\hline Secondary generalized convulsions & $6(11)$ \\
\hline \multicolumn{2}{|l|}{ Treatment strategy, $\mathrm{n}(\%)$} \\
\hline Pure lesionectomy & $5(10)$ \\
\hline Extended lesionectomy & $38(73)$ \\
\hline Anterior temporal lobectomy & $9(17)$ \\
\hline Additional amygdalohippocampectomy, n (\%) & $17(33)$ \\
\hline Favorable seizure outcome (ILAE class I), n (\%) & $47(90)$ \\
\hline
\end{tabular}

from CCM-related DRE and laterally in 18 patients. In the subgroup of patients with DRE and temporal lobe CCM, 2 patients $(6 \%)$ underwent pure lesionectomy, 27 patients (75\%) underwent extended lesionectomy, and 7 patients (19\%) underwent anterior temporal lobectomy. Additional amygdalohippocampectomy was performed in 14 patients (39\%) suffering from DRE due to the CCM located within the temporal lobe. Specifically, additional amygdalohippocampectomy was performed in 10 patients with DRE due to location of the CCM in the mesial temporal lobe structures, and in 4 patients due to the diagnosis of additional mesial temporal sclerosis as a potential secondary focus of epilepsy. In all 14 patients with DRE and additional amygdalohippocampectomy, pre- and postoperative neuropsychological assessment was obtained. Six of 14 patients with DRE and additional amygdalohippocampectomy had impaired baseline memory functions (6 verbal and 5 nonverbal). Postoperatively, 2 of the 6 impaired patients suffered from further decline of memory function (verbal and nonverbal).

Overall, $32(89 \%)$ of 36 patients who suffered from DRE related to temporal lobe CCM achieved excellent seizure outcome (ILAE class I).

\section{Discussion}

The present study depicts the presurgical epileptological workup and surgical management of patients with CCMs located within the temporal lobe suffering from CRE in a dedicated high-volume epilepsy and neurovascular surgery center. Contrary to the management of epileptogenic CCMs in general, surgical decision-making in patients with epileptogenic CCMs located within the temporal lobe specifically concerns whether to perform lesionectomy alone or the additional resection of mesiotemporal structures. ${ }^{17}$ Using close interdisciplinary consultation in these patients might facilitate favorable seizure outcome after resective epilepsy surgery.

\section{Mechanisms of CCM-Related Epilepsy}

CCMs are clusters of dilated sinusoids filled with blood and lined with endothelium without intervening parenchyma. ${ }^{1}$ Unlike other epileptogenic lesions, CCMs do not include functioning parenchyma. Different epileptogenic effects of CCMs on adjacent brain tissue have previously been discussed, such as iron deposition of blood breakdown products, cellular inflammation, and gliosis. ${ }^{1,20}$

\section{Surgical Treatment of CCM-Related Epilepsy}

Several studies have described the surgical management of epileptogenic CCM in general, ${ }^{10,12,19}$ but few studies have addressed the details of surgical management for CCMs according to their specific anatomical location. ${ }^{11,21}$ Surgical decision-making is challenging in CCMs located within the temporal lobe. Particularly in patients with temporal lobe CCMs and DRE, seizure outcome often depends on the decision of whether or not to additionally resect mesiotemporal structures. ${ }^{7,16}$

\section{Sporadic Epilepsy in Temporal Lobe CCM}

As many as $40 \%$ of patients with CCM suffer from new-onset or sporadic seizure at initial presentation. ${ }^{4}$ Due to the higher epileptogenic potential of the temporal lobe, CCMs located within the temporal lobe tend to have a higher incidence of seizure compared to other anatomical locations. ${ }^{12}$ In cases with sporadic epilepsy related to CCM, the first-line treatment is medical therapy.,13 Seizure control is usually feasible in patients suffering from sporadic seizure under the application of 1 selected drug. ${ }^{1}$ Nevertheless, seizure control is not the only factor influencing surgical decision-making in patients with manageable sporadic seizures related to CCM. An overall hemorrhage rate of $2.4 \%$ per patient-year was identified across several studies. ${ }^{3,8,9,14}$ Rehemorrhage correlates not only with the risk of change in seizure control, but also with an increased possibility of neurological deterioration. Therefore, prevention of rehemorrhage in patients with CCM represents a neurovascular-based rationale for resective epilepsy surgery in patients with CCMs and sporadic seizures. However, certain occupations of affected patients do not allow long-term antiepileptic medication or increased risk of seizure (such as professional drivers, construction workers, etc.). ${ }^{15,22}$ Therefore, the indication for surgical treatment in CCM-related sporadic seizures might be based on neurovascular as well as epileptological rationales. This circumstance highlights the necessity of close interdisciplinary consultation for patients with CCM-related sporadic epilepsy.

\section{DRE in Temporal Lobe CCM}

The presence of CCM in patients with intractable seizures is a challenging situation. Patients with seizures over a minimum period of 2 years despite adequate treatment 
fulfill the criteria for DRE. Patients with cavernoma-related DRE are candidates for resective epilepsy surgery of the identified cavernous malformation to improve seizure control. In the present series, seizure outcome in patients with CCMs within the temporal lobe and DRE was excellent (89\% ILAE class I). This is consistent with the results of previous studies, underlining the importance of epilepsy surgery in cases with CCM and DRE. ${ }^{11,19,21}$ Nevertheless, in patients with CCMs located within the temporal lobe, competing pathological findings might interfere with the standard surgical preference of pure lesionectomy. Several studies have highlighted the importance of additional resection of the hemosiderin rim to gain seizure control. ${ }^{2,5}$ On some occasions, extended lesionectomy might not be feasible due to the eloquent location of the cavernous lesion. ${ }^{6}$ To determine the exact surgical strategy, including the extent of resective epilepsy surgery, a thorough presurgical epileptological workup is essential in order to balance predicted seizure control against perioperative risks. In various cases, the presurgical epileptological workup might lead to the need for additional amygdalohippocampectomy to achieve favorable postoperative seizure outcome in patients with CRE and CCMs located within the temporal lobe. However, contemplating the resection of epileptogenic brain tissue leading to potential restraint of memory function should be weighed against seizure control by experienced specialists, emphasizing epileptological workup and decision-making in patients with temporal lobe CCMs and CRE in a designated high-volume epilepsy center.

\section{Limitations}

The present study has several limitations. The data were analyzed retrospectively, although the data were collected prospectively. Therefore, the study suffers from the shortcomings of a retrospective analysis. Only including patients with CCMs located within the temporal lobe led to a small study sample. Furthermore, the present data represent the experience of only a single tertiary epilepsy and neurovascular center. However, the standardized presurgical evaluation process and surgical management may outweigh some of these shortcomings. The results of the present study should be considered for further amplification of interdisciplinary collaboration in patients suffering from CCM-related epilepsy.

\section{Conclusions}

Favorable seizure outcome is achievable in a substantial number of patients with epileptogenic CCM located in the temporal lobe, even if patients suffered from drugresistant CRE. For adequate counseling and monitoring, patients with CRE should undergo a thorough pre- and postsurgical evaluation in dedicated epilepsy surgery programs.

\section{References}

1. Awad I, Jabbour P: Cerebral cavernous malformations and epilepsy. Neurosurg Focus 21(1):e7, 2006

2. Baumann CR, Schuknecht B, Lo Russo G, Cossu M, Citterio A, Andermann F, et al: Seizure outcome after resection of cavernous malformations is better when surrounding hemosiderin-stained brain also is removed. Epilepsia 47:563-566, 2006

3. Gross BA, Du R: Hemorrhage from cerebral cavernous malformations: a systematic pooled analysis. J Neurosurg 126:1079-1087, 2017

4. Gross BA, Lin N, Du R, Day AL: The natural history of intracranial cavernous malformations. Neurosurg Focus 30(6):E24, 2011

5. Hammen T, Romstöck J, Dörfler A, Kerling F, Buchfelder M, Stefan H: Prediction of postoperative outcome with special respect to removal of hemosiderin fringe: a study in patients with cavernous haemangiomas associated with symptomatic epilepsy. Seizure 16:248-253, 2007

6. Jeelani NU, Jindahra P, Tamber MS, Poon TL, Kabasele P, James-Galton M, et al: 'Hemispherical asymmetry in the Meyer's Loop': a prospective study of visual-field deficits in 105 cases undergoing anterior temporal lobe resection for epilepsy. J Neurol Neurosurg Psychiatry 81:985-991, 2010

7. Komotar RJ, Mikell CB, McKhann GM II: "Epilepsy surgery" versus lesionectomy in patients with seizures secondary to cavernous malformations. Clin Neurosurg 55:101107,2008

8. Kondziolka D, Lunsford LD, Kestle JR: The natural history of cerebral cavernous malformations. J Neurosurg 83:820 824,1995

9. Moriarity JL, Wetzel M, Clatterbuck RE, Javedan S, Sheppard JM, Hoenig-Rigamonti K, et al: The natural history of cavernous malformations: a prospective study of 68 patients. Neurosurgery 44:1166-1173, 1999

10. Mouchtouris N, Chalouhi N, Chitale A, Starke RM, Tjoumakaris SI, Rosenwasser RH, et al: Management of cerebral cavernous malformations: from diagnosis to treatment. ScientificWorldJournal 2015:808314, 2015

11. Paolini S, Morace R, Di Gennaro G, Picardi A, Grammaldo LG, Meldolesi GN, et al: Drug-resistant temporal lobe epilepsy due to cavernous malformations. Neurosurg Focus 21(1):e8, 2006

12. Rocamora R, Mader I, Zentner J, Schulze-Bonhage A: Epilepsy surgery in patients with multiple cerebral cavernous malformations. Seizure 18:241-245, 2009

13. Rosenow F, Alonso-Vanegas MA, Baumgartner C, Blümcke I, Carreño M, Gizewski ER, et al: Cavernoma-related epilepsy: review and recommendations for management-report of the Surgical Task Force of the ILAE Commission on Therapeutic Strategies. Epilepsia 54:2025-2035, 2013

14. Schneble HM, Soumare A, Hervé D, Bresson D, Guichard JP, Riant F, et al: Antithrombotic therapy and bleeding risk in a prospective cohort study of patients with cerebral cavernous malformations. Stroke 43:3196-3199, 2012

15. Schneider M, Güresir Á, Borger V, Hamed M, Rácz A, Vatter H, et al: Preoperative tumor-associated epilepsy in patients with supratentorial meningioma: factors influencing seizure outcome after meningioma surgery. J Neurosurg [epub ahead of print October 11, 2019; DOI: 10.3171/2019.7.JNS19455]

16. Upchurch K, Stern JM, Salamon N, Dewar S, Engel J Jr, Vinters HV, et al: Epileptogenic temporal cavernous malformations: operative strategies and postoperative seizure outcomes. Seizure 19:120-128, 2010

17. Vale FL, Vivas AC, Manwaring J, Schoenberg MR, Benbadis SR: Temporal lobe epilepsy and cavernous malformations: surgical strategies and long-term outcomes. Acta Neurochir (Wien) 157:1887-1895, 2015

18. Vernooij MW, Ikram MA, Tanghe HL, Vincent AJ, Hofman A, Krestin GP, et al: Incidental findings on brain MRI in the general population. N Engl J Med 357:1821-1828, 2007

19. von der Brelie C, Malter MP, Niehusmann P, Elger CE, von Lehe M, Schramm J: Surgical management and long-term 
seizure outcome after epilepsy surgery for different types of epilepsy associated with cerebral cavernous malformations. Epilepsia 54:1699-1706, 2013

20. Willmore LJ, Sypert GW, Munson JB: Recurrent seizures induced by cortical iron injection: a model of posttraumatic epilepsy. Ann Neurol 4:329-336, 1978

21. Yang PF, Pei JS, Jia YZ, Lin Q, Xiao H, Zhang TT, et al: Surgical management and long-term seizure outcome after surgery for temporal lobe epilepsy associated with cerebral cavernous malformations. World Neurosurg 110:e659e670, 2018

22. Zanello M, Goodden JR, Colle H, Wager M, Hamer PCW, Smits A, et al: Predictors of epileptic seizures and ability to work in supratentorial cavernous angioma located within eloquent brain areas. Neurosurgery 85:E702-E713, 2019

\section{Disclosures}

Prof. Surges reports receiving honoraria from Bial, Desitin, Eisai, LivaNova, Novartis, and UCB Pharma.

\section{Author Contributions}

Conception and design: Schuss. Acquisition of data: Schuss, Marx, Borger, Brandecker, Hadjiathanasiou, Hamed, Schneider. Analysis and interpretation of data: Schuss, Marx, Borger, Hadjiathanasiou, Schneider, E Güresir. Drafting the article: Schuss, E Güresir. Critically revising the article: Borger, A Güresir, Schneider, Surges, Vatter, E Güresir. Reviewed submitted version of manuscript: Schuss, Borger, Brandecker, A Güresir, Hadjiathanasiou, Hamed, Schneider, Surges, Vatter, E Güresir. Statistical analysis: Schuss, Marx, Schneider,

E Güresir. Administrative/technical/material support: A Güresir. Study supervision: Schuss, Vatter, E Güresir.

\section{Correspondence}

Patrick Schuss: Rheinische Friedrich-Wilhelms-University, Bonn, Germany.patrick.schuss@ukbonn.de. 